\title{
छூ \\ Microscopic mechanisms for the Fermi-liquid behavior of Nb-doped strontium titanate
}

\author{
S. N. Klimin* and J. Tempere ${ }^{\dagger}$ \\ Theorie van Kwantumsystemen en Complexe Systemen (TQC), Universiteit Antwerpen, Universiteitsplein 1, B-2610 Antwerpen, Belgium \\ D. van der Marel \\ Département de Physique de la Matière Condensée, Université de Genève, CH-1211 Genève 4, Switzerland
}

J. T. Devreese

Theorie van Kwantumsystemen en Complexe Systemen (TQC), Universiteit Antwerpen, Universiteitsplein 1, B-2610 Antwerpen, Belgium

(Received 4 June 2012; published 13 July 2012)

\begin{abstract}
The relaxation rate in $\mathrm{Nb}$-doped strontium titanate involving different scattering channels is investigated theoretically. It is demonstrated that the total relaxation rate in $\operatorname{SrTi}_{1-x} \mathrm{Nb}_{x} \mathrm{O}_{3}$ is provided mainly by two mechanisms. The Baber electron-electron scattering with participation of both Coulomb and phonon-mediated electron-electron interactions provides the $T^{2}$ dependence of the relaxation rate. The scattering on the potential landscape caused by impurities is responsible for the residual relaxation rate at low temperatures. A good agreement with experiment is achieved accounting for all phonon branches in strontium titanate, both the optical and acoustic phonons. It is shown that the effective electron-electron interaction can be attractive in strontium titanate, and provides superconductivity at low temperatures and Fermi-liquid response in a wide range of temperatures. Thus our microscopic model supports the notion that superconductivity and Fermi-liquid properties of $n$-type $\mathrm{SrTiO}_{3}$ have a common origin.
\end{abstract}

DOI: 10.1103/PhysRevB.86.045113

PACS number(s): 71.10.Ay, 71.38.Fp, 74.20.-z, 72.10.Bg

\section{INTRODUCTION}

In Ref. 1, a common origin of the Fermi-liquid properties and superconductivity in $\mathrm{Nb}$-doped strontium titanate has been postulated and induced from the phenomenological treatment of the resistivity data and superconducting critical temperatures $T_{c}$. In the present work, we deduce the temperature dependence of the resistivity and the superconducting $T_{c}$ 's from microscopic model calculations. The relaxation rate in $\mathrm{SrTi}_{1-x} \mathrm{Nb}_{x} \mathrm{O}_{3}$ can be accounted for by various scattering mechanisms. The Fermi-liquid properties of a charge carrier gas in a crystal are provided by the effective electron-electron interaction. This interaction is an interplay of the Coulomb repulsion and the phonon-mediated attraction provided by the Fröhlich electron-phonon interaction with the optical phonons. Strontium titanate is a strongly polar crystal with static dielectric constant $\varepsilon_{0} \approx 240$ and high-frequency dielectric constant $\varepsilon_{\infty} \approx 5.44$. $^{2}$ The ratio $\eta=\varepsilon_{\infty} / \varepsilon_{0}$ is very small in strontium titanate. Due to a small $\eta$, the Coulomb repulsion and the optical phonon-mediated attraction significantly compensate each other. Thus the contribution of the other electronphonon interactions, e.g., the deformation interaction with the acoustic phonons, can be important for the sign of the total effective electron-electron interaction. The fact that $n$-doped $\mathrm{SrTiO}_{3}$ is a superconductor ${ }^{3,4}$ implies that in this material the phonon-mediated attraction due to both optical and acoustic phonons can overcome the Coulomb repulsion. This yields superconductivity in strontium titanate at low temperatures about $T \sim 1 \mathrm{~K}$. In this connection, we verify the suggestion ${ }^{1}$ that the dc resistivity and the relaxation rate in $\mathrm{Nb}$-doped $\mathrm{SrTiO}_{3}$ can be due to the effective electron-electron interaction. There are two channels for the Fermi-liquid response of metals and strongly doped semiconductors with complex conductivity bands due to the electron-electron interaction: the normal and umklapp $^{5-7}$ scattering processes.
Several other scattering mechanisms besides the effective electron-electron interaction are present in strontium titanate. For completeness, they must be included in the treatment, as far as they may contribute to the total resistivity. In the present work, the following additional scattering mechanisms are considered.

(1) The electron-phonon interaction can contribute to the effective electron-electron scattering as mentioned above. Besides this, the direct scattering of the electrons by the LO phonons can contribute to the resistivity of the polar crystals in the same way as to the optical absorption. $2,8,9$

(2) In the context of small polarons, the electron-phonon scattering results in a temperature dependence of the resistivity which is close to the $T^{2}$ behavior. ${ }^{10}$ Therefore the electronLO-phonon scattering may influence the Fermi-liquid-like temperature dependence of the dc response of the charge carriers.

As established in Refs. 2 and 8, the polarons in strontium titanate are large (Fröhlich) polarons rather than the small polarons treated in Ref. 10. In this connection, the electronLO-phonon scattering is considered here in terms of the large polarons.

(3) Finally, the scattering of the electrons on the potential landscape induced by the impurities (for example, the $\mathrm{Nb}$ donors) can bring a nonnegligible contribution to the total relaxation rate and resistivity, especially in the low-temperature regime.

\section{THEORY AND RESULTS}

\section{A. Baber scattering}

Within a parabolic model for a simple conductivity band, the electron-electron interaction due to the normal (i.e., within the first Brillouin zone) scattering processes does not 
contribute to the carrier electric response owing to momentum conservation, even for an anisotropic band. ${ }^{11}$ However, for a nonparabolic and/or complex conductivity band with, e.g., light and heavy carriers, the carrier response can be nonzero as first found by Baber. ${ }^{12,13}$ Thus the resistivity can be provided by the interaction between charge carriers with different band masses or between electrons and holes.

The Baber scattering mechanism is the most promising candidate to provide the main contribution to the total relaxation rate of $\mathrm{Nb}$-doped strontium titanate. We calculate the resistivity using the Boltzmann equation. Further on, we follow the notations of Ref. 14. The nonequilibrium distribution function $f_{n, \mathbf{k}}$ for the carriers in the $n$th subband is determined as

$$
f_{n, \mathbf{k}}=f_{n, \mathbf{k}}^{(0)}-\Phi_{n, \mathbf{k}} \frac{\partial f_{n, \mathbf{k}}^{(0)}}{\partial \varepsilon_{n, \mathbf{k}}},
$$

where $f_{n, \mathbf{k}}^{(0)} \equiv f\left(\varepsilon_{n, \mathbf{k}}\right)$ is the equilibrium electron distribution function in the $n$th subband of the conduction band,

$$
f\left(\varepsilon_{n, \mathbf{k}}\right)=\frac{1}{e^{\left(\varepsilon_{n, \mathbf{k}}-\mu\right) / k_{B} T}+1},
$$

and $\varepsilon_{n, \mathbf{k}}$ is the electron energy.

The function $\Phi_{n, \mathbf{k}}$ is a measure of the deviation from equilibrium in the electron distribution. The inner product of two real functions $\Psi$ and $\Phi$ is defined by $\langle\Psi, \Phi\rangle \equiv$ $\sum_{n} \int d \mathbf{k} \Psi_{n, \mathbf{k}} \Phi_{n, \mathbf{k}}$. The Boltzmann equation can be written as

$$
X=P \Phi,
$$

where $P$ is the scattering operator which transforms the function $\Phi$ into another function $\Psi=P \Phi$, and $X$ represents the left-hand side of the Boltzmann equation,

$$
X=-\mathbf{v}_{n, \mathbf{k}} \cdot \frac{\partial f\left(\varepsilon_{n, \mathbf{k}}\right)}{\partial T} \nabla T-\mathbf{v}_{n, \mathbf{k}} \cdot e \frac{\partial f\left(\varepsilon_{n, \mathbf{k}}\right)}{\partial \varepsilon_{n, \mathbf{k}}} \mathbf{E} .
$$

Here, $\mathbf{v}_{n, \mathbf{k}}$ is the velocity in the $n$th subband,

$$
\mathbf{v}_{n, \mathbf{k}}=\frac{1}{\hbar} \frac{\partial \varepsilon_{n, \mathbf{k}}}{\partial \mathbf{k}} .
$$

The collision integral of the Boltzmann equation can be represented as $\langle\Phi, P \Phi\rangle$. The Boltzmann equation in the form (3) implies that

$$
\langle\Phi, X\rangle=\langle\Phi, P \Phi\rangle .
$$

The variational principle established by $\operatorname{Ziman}^{14}$ states that the solution of the Boltzmann equation gives $\langle\Phi, P \Phi\rangle$ its maximum value. Thus $\Phi_{n, \mathbf{k}}$ can be approximated by a trial variational function.

In the notations of Ref. 14, the dc resistivity is expressed by the formula

$$
\rho=\frac{\langle\Phi, P \Phi\rangle}{[\langle\Phi, X(E=1)\rangle]^{2}},
$$

where $X(E=1)$ represents the left-hand side of the Boltzmann equation in a unit electric field $E=1$ (and in the absence of the temperature gradients). The normalization factor is determined by the expression

$$
\langle\Phi, X(E=1)\rangle=2 \sum_{n} \int e \mathbf{v}_{n, \mathbf{k}} \Phi_{n, \mathbf{k}} \frac{\partial f\left(\varepsilon_{n, \mathbf{k}}\right)}{\partial \varepsilon_{n, \mathbf{k}}} \frac{d \mathbf{k}}{(2 \pi)^{3}} .
$$

The collision integral for the electron-electron scattering processes is

$$
\begin{aligned}
\langle\Phi, P \Phi\rangle= & \frac{1}{2 k_{B} T} \sum_{n, n^{\prime}} \int \frac{d \mathbf{k}_{1}}{(2 \pi)^{3}} \frac{d \mathbf{k}_{2}}{(2 \pi)^{3}} \frac{d \mathbf{k}_{3}}{(2 \pi)^{3}} \frac{d \mathbf{k}_{4}}{(2 \pi)^{3}} \\
& \times\left(\Phi_{n, 1}+\Phi_{n^{\prime}, 2}-\Phi_{n, 3}-\Phi_{n^{\prime}, 4}\right)^{2} \\
& \times \mathcal{P}\left(n, 1, n^{\prime}, 2 \rightarrow n, 3, n^{\prime}, 4\right) .
\end{aligned}
$$

Here, $\mathcal{P}\left(n, 1, n^{\prime}, 2 \rightarrow n, 3, n^{\prime}, 4\right)$ is the scattering probability,

$$
\begin{aligned}
\mathcal{P}\left(n, 1, n^{\prime}, 2 \rightarrow n, 3, n^{\prime}, 4\right) \\
=\left(U_{\mathbf{k}_{1}, \mathbf{k}_{3}}^{(\mathrm{eff})}\right)^{2} f\left(\varepsilon_{n, \mathbf{k}_{1}}\right) f\left(\varepsilon_{n^{\prime}, \mathbf{k}_{2}}\right)\left[1-f\left(\varepsilon_{n, \mathbf{k}_{3}}\right)\right]\left[1-f\left(\varepsilon_{n^{\prime}, \mathbf{k}_{4}}\right)\right] \\
\quad \times \frac{2 \pi}{\hbar} \delta\left(\varepsilon_{n, \mathbf{k}_{1}}+\varepsilon_{n^{\prime}, \mathbf{k}_{2}}-\varepsilon_{n, \mathbf{k}_{3}}-\varepsilon_{n^{\prime}, \mathbf{k}_{4}}\right) \\
\quad \times(2 \pi)^{3} \delta\left(\mathbf{k}_{1}+\mathbf{k}_{2}-\mathbf{k}_{3}-\mathbf{k}_{4}\right),
\end{aligned}
$$

with $U_{\mathbf{k}_{1}, \mathbf{k}_{3}}^{(\text {eff }}$ the matrix element of the effective electronelectron interaction, which includes both the Coulomb repulsion and the phonon-mediated attraction.

The trial variational function $\Phi_{n, \mathbf{k}}$ is chosen in the form ${ }^{14}$

$$
\Phi_{n, \mathbf{k}}=\mathbf{v}_{n, \mathbf{k}} \cdot \mathbf{u},
$$

where $\mathbf{u}$ is the unit vector parallel to the applied electric field. Thus the collision integral is

$$
\begin{aligned}
\langle\Phi, P \Phi\rangle= & \frac{1}{2 k_{B} T} \frac{1}{(2 \pi)^{9}} \frac{2 \pi}{\hbar} \sum_{n, n^{\prime}} \int d \mathbf{k}_{1} d \mathbf{k}_{2} d \mathbf{k}_{3} d \mathbf{k}_{4} \\
& \times\left[\left(\mathbf{v}_{n, \mathbf{k}_{1}}+\mathbf{v}_{n^{\prime}, \mathbf{k}_{2}}-\mathbf{v}_{n, \mathbf{k}_{3}}-\mathbf{v}_{n^{\prime}, \mathbf{k}_{4}}\right) \cdot \mathbf{u}\right]^{2} \\
& \times\left(U_{\mathbf{k}_{1}, \mathbf{k}_{3}}^{\text {(eff) }}\right)^{2} f\left(\varepsilon_{n, \mathbf{k}_{1}}\right) f\left(\varepsilon_{n^{\prime}, \mathbf{k}_{2}}\right)\left[1-f\left(\varepsilon_{n, \mathbf{k}_{3}}\right)\right] \\
& \times\left[1-f\left(\varepsilon_{n^{\prime}, \mathbf{k}_{4}}\right)\right] \delta\left(\varepsilon_{n, \mathbf{k}_{1}}+\varepsilon_{n^{\prime}, \mathbf{k}_{2}}-\varepsilon_{n, \mathbf{k}_{3}}-\varepsilon_{n^{\prime}, \mathbf{k}_{4}}\right) \\
& \times \delta\left(\mathbf{k}_{1}+\mathbf{k}_{2}-\mathbf{k}_{3}-\mathbf{k}_{4}\right) .
\end{aligned}
$$

The electrons in the conduction band are described by the matrix Hamiltonian from Ref. 1:

$$
H=4\left(\begin{array}{ccc}
\varepsilon_{1}(\mathbf{k}) & 0 & 0 \\
0 & \varepsilon_{2}(\mathbf{k}) & 0 \\
0 & 0 & \varepsilon_{3}(\mathbf{k})
\end{array}\right)+\frac{1}{2} W,
$$

with the energies

$$
\begin{aligned}
& \varepsilon_{1}=t_{\delta} \sin ^{2}\left(\frac{a_{0} k_{x}}{2}\right)+t_{\pi} \sin ^{2}\left(\frac{a_{0} k_{y}}{2}\right)+t_{\pi} \sin ^{2}\left(\frac{a_{0} k_{z}}{2}\right), \\
& \varepsilon_{2}=t_{\pi} \sin ^{2}\left(\frac{a_{0} k_{x}}{2}\right)+t_{\delta} \sin ^{2}\left(\frac{a_{0} k_{y}}{2}\right)+t_{\pi} \sin ^{2}\left(\frac{a_{0} k_{z}}{2}\right), \\
& \varepsilon_{3}=t_{\pi} \sin ^{2}\left(\frac{a_{0} k_{x}}{2}\right)+t_{\pi} \sin ^{2}\left(\frac{a_{0} k_{y}}{2}\right)+t_{\delta} \sin ^{2}\left(\frac{a_{0} k_{z}}{2}\right),
\end{aligned}
$$

where $a_{0}$ is the lattice constant. The matrix $W$

$$
W=\left(\begin{array}{ccc}
2 D & \xi & \xi \\
\xi & 2 D & \xi \\
\xi & \xi & -4 D
\end{array}\right)
$$

describes the mixing of subbands within the conductivity band. We use the values of the band parameters $t_{\delta}, t_{\pi}, D, \xi$ 
from Ref. 1: $t_{\delta}=35 \mathrm{meV}, t_{\pi}=615 \mathrm{meV}, \xi=18.8 \mathrm{meV}$, and $D=2.2 \mathrm{meV}$.

In the present treatment, we neglect the band mixing because it is relatively small, and assume the anisotropic parabolic dispersion in each of three subbands of the conductivity band (as in Refs. 1 and 2) with the tensor effective masses $\left|m_{j j}^{(n)}\right|(j=x, y, z, n=1,2,3)$. The masses $m_{j j}^{(n)}$ can take two values: the "light" mass $m_{\pi}=2 \hbar^{2} / a_{0}^{2} t_{\pi}$ and the "heavy" mass $m_{\delta}=2 \hbar^{2} / a_{0}^{2} t_{\delta}$. Using the above band parameters of strontium titanate and the lattice constant $a_{0}=3.905 \AA$, we arrive at the band masses $m_{\pi} \approx 1.6 m_{e}$ and $m_{\delta} \approx 29 m_{e}$, where $m_{e}$ is the electron mass in vacuum.

The effective electron-electron interaction in $\mathrm{SrTiO}_{3}$ is considered in the following way. In the standard diagram technique, the effective interaction corresponding to an elementary electron-electron scattering process for a single-branch electron-phonon system is given by the expression $^{15}$

$$
U_{\mathbf{k}, \mathbf{k}^{\prime}}^{(\mathrm{eff})} \equiv U_{e-e}^{(\mathrm{eff})}(\mathbf{q}, \omega)=U_{C}(\mathbf{q})+\frac{1}{\hbar}\left|V_{\mathbf{q}}\right|^{2} \mathcal{D}^{(0)}(\mathbf{q}, \omega),
$$

where $\mathcal{D}^{(0)}(\mathbf{q}, \omega)$ is the free-phonon Green's function,

$$
\mathcal{D}^{(0)}(\mathbf{q}, \omega)=-\frac{2 \omega_{\mathbf{q}}}{\omega_{\mathbf{q}}^{2}-\omega^{2}},
$$

$\mathbf{q}$ is the momentum transfer $\mathbf{q}=\mathbf{k}^{\prime}-\mathbf{k}$, and $\omega$ is equal to the transition frequency $\omega=\left(\varepsilon_{n^{\prime}, \mathbf{k}^{\prime}}-\varepsilon_{n, \mathbf{k}}\right) / \hbar$ (where $n, n^{\prime}$ are the indices of the subbands of the conduction band). Thus the effective interaction is

$$
U_{e-e}^{(\mathrm{eff})}(\mathbf{q}, \omega)=U_{C}(\mathbf{q})-\left|V_{\mathbf{q}}\right|^{2} \frac{2 \hbar \omega_{\mathbf{q}}}{\hbar^{2} \omega_{\mathbf{q}}^{2}-\left(\varepsilon_{n^{\prime}, \mathbf{k}^{\prime}}-\varepsilon_{n, \mathbf{k}}\right)^{2}} .
$$

The effective interaction (15) is written for a one-branch phonon system and does not account for screening. For our calculations, both the screening and the multibranch phonon system must be accounted for. The extension of the above formulas to the multibranch phonon system is straightforward:

$$
\begin{aligned}
U_{e-e}^{(\text {eff })}(\mathbf{q}, \omega)= & U_{C}(\mathbf{q})-\frac{1}{\hbar} \sum_{\lambda} \frac{2 \omega_{\mathbf{q}, \lambda}\left|V_{\mathbf{q}, \lambda}\right|^{2}}{\omega_{\mathbf{q}, \lambda}^{2}-\omega^{2}} \\
& -\frac{1}{\hbar} \frac{2 \omega_{\mathbf{q}}^{(a c)}\left|V_{\mathbf{q}}^{(a c)}\right|^{2}}{\left(\omega_{\mathbf{q}}^{(a c)}\right)^{2}-\omega^{2}}
\end{aligned}
$$

where $\lambda$ labels different optical-phonon branches. The contribution to the effective interaction due to the acoustic phonons (with the interaction amplitudes $V_{\mathbf{q}}^{(a c)}$ ) is written here as a separate term. The screening for the Coulomb interaction and for the phonon-mediated interaction can be introduced here as in Ref. 15,

$$
\begin{aligned}
U_{e-e}^{(\text {eff })}(\mathbf{q}, \omega)= & \frac{4 \pi e^{2}}{q^{2} \varepsilon_{\infty} \epsilon_{e}(\mathbf{q}, \omega)}-\frac{1}{\hbar} \sum_{\lambda} \frac{\left|V_{\mathbf{q}, \lambda}\right|^{2}}{\left[\epsilon_{e}(\mathbf{q}, \omega)\right]^{2}} \frac{2 \omega_{\mathbf{q}, \lambda}}{\omega_{\mathbf{q}, \lambda}^{2}-\omega^{2}} \\
& -\frac{1}{\hbar} \frac{2 \omega_{\mathbf{q}}^{(a c)}\left|V_{\mathbf{q}}^{(a c)}\right|^{2}}{\left(\omega_{\mathbf{q}}^{(a c)}\right)^{2}-\omega^{2}}
\end{aligned}
$$

where $\epsilon_{e}(\mathbf{q}, \omega)$ is the electron screening factor. Note that screening is different for the Coulomb and phonon-mediated interactions, and that the contribution due to the acoustic phonons is not screened.

In the experimental situation of Ref. 1, the Fermi energy of the electrons in the conduction band substantially exceeds their thermal energy $k_{B} T$. Therefore only the electrons in a thermal layer near the Fermi surface bring a dominating contribution to the relaxation rate. In other words, the energies of the relevant electrons are close to the Fermi energy, and the difference $\varepsilon_{n^{\prime}, \mathbf{k}^{\prime}}-\varepsilon_{n, \mathbf{k}} \sim k_{B} T$ is relatively small with respect to the Fermi energy.

The major contribution to the collision integral (10) lies in the range of the momentum transfer $q \propto 2 k_{F}$, where $k_{F}$ is the electron wave vector at the Fermi surface (in general, angle-dependent due to the band anisotropy). In $\mathrm{SrTiO}_{3}$, the corresponding acoustic-phonon energy satisfies the condition $\hbar \omega_{\mathbf{q}}^{(a c)} \gtrsim k_{B} T$ in the range $T<100 \mathrm{~K}$ for all samples treated in the experiment ${ }^{1}$ except the lowest-doped sample, for which $\hbar \omega_{\mathbf{q}}^{(a c)} / k_{B} T \sim 1$ at $T=100 \mathrm{~K}$. For the lower temperatures and/or for the higher doping contents the aforesaid condition is fulfilled. The LO-phonon energies in $\mathrm{SrTiO}_{3}$ are much higher than the thermal energy in the considered temperature range. Thus, owing to the fact that the electron-electron scattering effectively occurs close to the Fermi surface, retardation effects in the phonon-mediated interaction can be neglected. Therefore we can approximate the effective interaction by the expression

$$
U_{e-e}^{(\mathrm{eff})}(\mathbf{q})=\frac{4 \pi e^{2}}{q^{2} \varepsilon_{\infty} \epsilon_{e}(\mathbf{q})}-\sum_{\lambda} \frac{2\left|V_{\mathbf{q}, \lambda}\right|^{2}}{\hbar \omega_{\mathbf{q}, \lambda}\left[\epsilon_{e}(\mathbf{q})\right]^{2}}-\frac{2\left|V_{\mathbf{q}}^{(a c)}\right|^{2}}{\hbar \omega_{\mathbf{q}}^{(a c)}},
$$

where $\epsilon_{e}(\mathbf{q})$ is the static electron screening factor. The different screening of the Coulomb and phonon-mediated interactions, however, can strongly influence the resulting interaction. In the present calculation, the Thomas-Fermi (TF) screening factor is used in the effective interaction:

$$
\epsilon_{e}(\mathbf{q})=1+\frac{\kappa_{s}^{2}}{q^{2}}
$$

where $\kappa_{s}$ is the inverse TF screening length,

$$
\kappa_{s}=\left(\frac{6 \pi e^{2} n_{0}}{\varepsilon_{\infty} E_{F}}\right)^{1 / 2},
$$

$n_{0}$ is the carrier density, and $E_{F}$ is the Fermi energy. Note that $\epsilon_{e}(\mathbf{q})$ is the static screening factor for the electron gas (without the lattice polarization). Therefore $\kappa_{s}$ contains the high-frequency dielectric constant $\varepsilon_{\infty}$ rather than the static dielectric constant $\varepsilon_{0}$. The material parameters for the Fröhlich interaction with the optical phonons are taken the same as in Ref. 2. The values of the TO- and LO-phonon frequencies, the actual electron densities, and the plasma frequencies $\omega_{p}$ determined from the experiment ${ }^{8}$ are represented in Table I.

For the acoustic-phonon contribution, we use the interaction amplitudes for the deformation potential from Ref. 16:

$$
V_{\mathbf{q}}^{(a c)}=\left(4 \pi \alpha_{a c}\right)^{1 / 2} \frac{\hbar^{2}}{m_{D}} q^{1 / 2}
$$


TABLE I. Optical-phonon frequencies, electron densities, and plasma frequencies of doped strontium titanate.

\begin{tabular}{|c|c|c|c|c|c|c|c|c|}
\hline & $\begin{array}{c}x=0.1 \% \\
T=7 \mathrm{~K}\end{array}$ & $\begin{aligned} x & =0.1 \% \\
T & =300 \mathrm{~K}\end{aligned}$ & $\begin{array}{c}x=0.2 \% \\
T=7 \mathrm{~K}\end{array}$ & $\begin{aligned} x & =0.2 \% \\
T & =300 \mathrm{~K}\end{aligned}$ & $\begin{array}{c}x=0.9 \% \\
T=7 \mathrm{~K}\end{array}$ & $\begin{aligned} x & =0.9 \% \\
T & =300 \mathrm{~K}\end{aligned}$ & $\begin{array}{l}x=2 \% \\
T=7 \mathrm{~K}\end{array}$ & $\begin{array}{c}x=2 \% \\
T=300 \mathrm{~K}\end{array}$ \\
\hline$\hbar \omega_{\mathrm{TO}, 1}(\mathrm{meV})$ & 2.27 & 11.5 & 2.63 & 11.5 & 6.01 & 12.1 & 8.51 & 13.0 \\
\hline$\hbar \omega_{\mathrm{LO}, 1}(\mathrm{meV})$ & 21.2 & 21.2 & 21.2 & 21.2 & 21.2 & 21.2 & 21.2 & 21.2 \\
\hline$\hbar \omega_{\mathrm{TO}, 2}(\mathrm{meV})$ & 21.2 & 21.8 & 21.2 & 21.8 & 21.2 & 21.8 & 21.2 & 21.8 \\
\hline$\hbar \omega_{\mathrm{LO}, 2}(\mathrm{meV})$ & 58.4 & 58.4 & 58.4 & 58.4 & 58.4 & 58.4 & 58.4 & 58.4 \\
\hline$\hbar \omega_{\mathrm{TO}, 3}(\mathrm{meV})$ & 67.6 & 67.1 & 67.6 & 67.1 & 67.6 & 67.1 & 67.6 & 67.1 \\
\hline$\hbar \omega_{\mathrm{LO}, 3}(\mathrm{meV})$ & 98.7 & 98.7 & 98.7 & 98.7 & 98.7 & 98.7 & 98.7 & 98.7 \\
\hline$n_{0}\left(\mathrm{~cm}^{-3}\right)$ & $1.7 \times 10^{19}$ & $1.7 \times 10^{19}$ & $3.4 \times 10^{19}$ & $3.4 \times 10^{19}$ & $1.5 \times 10^{20}$ & $1.5 \times 10^{20}$ & $3.4 \times 10^{20}$ & $3.4 \times 10^{20}$ \\
\hline$\hbar \omega_{p}(\mathrm{eV})$ & 0.1 & 0.1 & 0.14 & 0.14 & 0.29 & 0.29 & 0.44 & 0.44 \\
\hline
\end{tabular}

with the dimensionless coupling constant

$$
\alpha_{a c}=\frac{E_{d}^{2} m_{D}^{2}}{8 \pi n \hbar^{3} v}
$$

where $n$ is the density, $E_{d}$ is the deformation potential, $v$ is the sound velocity, and $m_{D}$ is the density-of-state band mass. It is worth noting that the acoustic-phonon interaction amplitude (21) in fact does not depend on the mass $m_{D}$. The sound velocity in strontium titanate is taken $v \approx 8.1 \times 10^{3} \mathrm{~m} \mathrm{~s}^{-1} .{ }^{17}$

The relaxation rate is determined using its relation to the resistivity,

$$
\frac{1}{\tau_{\text {eff }}}=\frac{\omega_{p}^{2}}{4 \pi} \rho,
$$

where $\omega_{p}$ is the plasma frequency for the electron gas in the conductivity band determined in the same way as in Ref. 1, and listed in Table I. In Fig. 1, we represent the numerical results for the relaxation rate provided by Baber scattering as a function of temperature for the actual electron densities, which are determined from the experimental values of the doping level given in Ref. 1. We possess the measured values for all material parameters of strontium titanate, except the deformation potential $E_{d}$. In this connection, the value

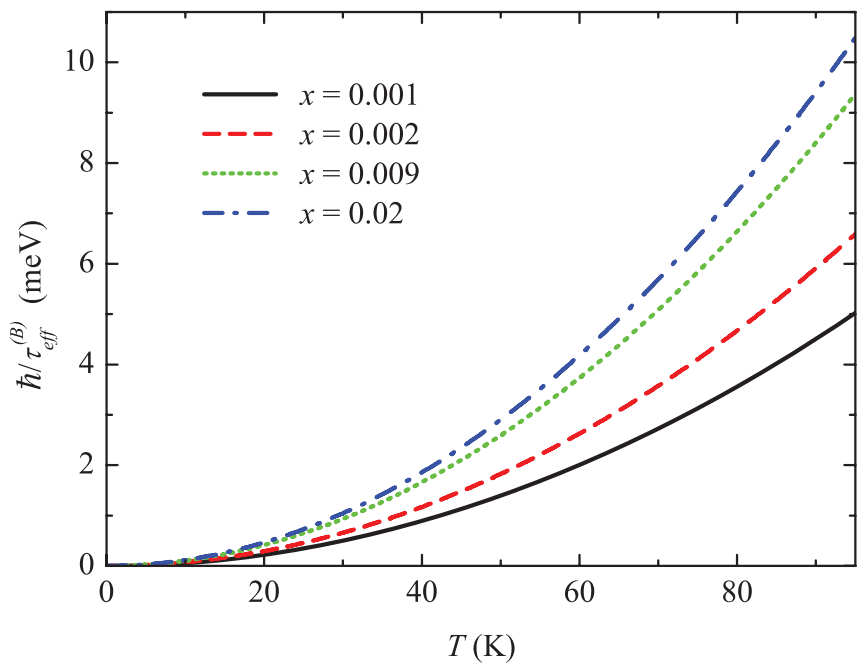

FIG. 1. (Color online) Relaxation rate provided by the Baber scattering in $\mathrm{Nb}$-doped $\mathrm{SrTiO}_{3}$ as a function of the temperature for the actual electron densities corresponding to the experimental values of the doping from Ref. 1.
$E_{d}=23.3 \mathrm{eV}$ has been chosen to fit the $T^{2}$ dependence of the relaxation rate to the experimental data for the lowest doping level $x=0.1 \%$. For the other densities, we keep one and the same value of $E_{d}$. Typical values of the deformation potential in other crystals found in the literature are about 10 to $30 \mathrm{eV}$, so that the chosen value is realistic.

The relaxation rates calculated with the same value of $E_{d}$ for all densities reproduce the density dependence of the experimentally obtained relaxation rate fairly well, except a residual background contribution which does not vanish when the temperature tends to zero. Therefore the suggestion ${ }^{1}$ that the $T^{2}$ dependence of the DC resistivity in $\operatorname{SrTi}_{1-x} \mathrm{Nb}_{x} \mathrm{O}_{3}$ can be provided by the Baber scattering mechanism is supported by the present calculation.

When both optical and acoustic phonons are taken into account, the effective electron-electron interaction at the Fermi surface can become attractive. With optical phonons only (i.e., neglecting acoustic-phonon contribution) this attraction is not possible. Moreover, the effective electron-electron interaction without participation of the acoustic phonons is approximately Coulomb-like. The relaxation rate for a Coulomb-like electron-electron interaction monotonically decreases with an increasing density. The density dependence of the relaxation rate observed experimentally can be explained by a relative increase of the attraction provided by the acoustic phonons with respect to the Coulomb repulsion and the interaction due to the optical phonons. The latter two become more strongly screened when the density rises, while the deformation potential is not screened. The increasing density dependence of the relaxation rate therefore supports our hypothesis that the phonon-mediated attraction (provided by both optical and acoustic phonons) in strontium titanate can overcome the Coulomb repulsion even in the normal phase - at sufficiently high temperatures, when superconductivity does not exist.

\section{B. Other mechanisms}

We suggest that the relatively small residual relaxation rate in the $\mathrm{Nb}$-doped $\mathrm{SrTiO}_{3}$ can be accounted for by the scattering of the electrons on the potential landscape created by the impurities. The contribution to the dc resistivity due to the scattering of the electrons by the potential of the impurities is calculated using the approximation of the time-dependent relaxation time within the Boltzmann equation approach. Assuming the impurities chaotically distributed in space, the DC conductivity tensor is determined by the expression (see 
Ref. 18)

$$
\sigma_{i j}=-\frac{2 e^{2}}{\hbar^{3}} \sum_{n} \int \frac{d \mathbf{k}}{(2 \pi)^{3}} \tau_{n}(\mathbf{k})\left(\frac{\partial f_{0}\left(\varepsilon_{n, \mathbf{k}}\right)}{\partial \varepsilon_{n, \mathbf{k}}}\right)\left(\mathbf{v}_{n, \mathbf{k}}\right)_{i}\left(\mathbf{v}_{n, \mathbf{k}}\right)_{j},
$$

where $\left(\mathbf{v}_{n, \mathbf{k}}\right)_{j}$ are the components of the electron velocity in the conduction band.

For the relaxation time $\tau_{n}(\mathbf{k})$, we apply the approximation of the isotropic threefold degenerate conduction band with the effective density-of-states band mass $m_{D} \equiv\left(m_{x x}^{(n)} m_{y y}^{(n)} m_{z z}^{(n)}\right)^{1 / 3}$. In this approximation, $\tau_{n}(\mathbf{k})=\tau(\mathbf{k})$, and the conductivity tensor is reduced to the scalar expression $\sigma \equiv \rho^{-1}$,

$$
\frac{1}{\rho}=-\left.2 \frac{e^{2}}{\hbar m_{D}^{2}} \int \frac{d \mathbf{k}}{(2 \pi)^{3}} k^{2} \tau(\mathbf{k})\left(\frac{\partial f_{0}(\varepsilon)}{\partial \varepsilon}\right)\right|_{\varepsilon=\varepsilon(\mathbf{k})},
$$

where $\rho$ is the dc resistivity. The momentum-dependent relaxation time $\tau(\mathbf{k})$ for the chaotically distributed impurities with the density $n_{I}$ is determined using the differential scattering cross-section $\sigma(k, \theta)$ as ${ }^{18}$

$$
\frac{1}{\tau(k)}=2 \pi n_{I} v_{\mathbf{k}} \int_{0}^{\pi} \sigma(k, \theta)(1-\cos \theta) d \theta .
$$

The scattering cross-section $\sigma(k, \theta)$ is calculated here in the Born approximation ${ }^{19}$

$$
\sigma(k, \theta)=\frac{m_{D}^{2}}{4 \pi^{2} \hbar^{4}}\left|\int U(\mathbf{r}) e^{-i \mathbf{q} \cdot \mathbf{r}} d \mathbf{r}\right|^{2},
$$

where $U(\mathbf{r})$ is the potential created by an impurity, and $\mathbf{q}=$ $\mathbf{k}^{\prime}-\mathbf{k}$ is the momentum transfer (with $k^{\prime}=k$, because the scattering is elastic).

To the best of our knowledge, the true potentials created by the impurities in doped strontium titanate are not yet reliably known. We suggest that the majority contribution to the impurity scattering is due to the ionized niobium donors. Thus we assume that the density $n_{I} \approx n_{0}$ (where $n_{0}$ is the electron density), and that the potential created by an ion can be modeled by a pseudopotential corresponding to the Yukawa (screened Coulomb) potential:

$$
U(\mathbf{r})=-\frac{U_{0}}{r} \exp \left(-\kappa_{I} r\right)
$$

where $U_{0}=e^{2} / \varepsilon_{0} ; \kappa_{I}$ is the inverse screening length for the impurity potential. The scattering cross-section $\sigma(k, \theta)$ with the potential (28) is

$$
\sigma(k, \theta)=\left(\frac{2 m_{D} U_{0}}{\hbar^{2}}\right)^{2} \frac{1}{\left[2 k^{2}(1-\cos \theta)+\kappa_{I}^{2}\right]^{2}} .
$$

Integrating in Eq. (26) with (29), the inverse momentumdependent relaxation time is obtained to be

$$
\frac{1}{\tau(k)}=\frac{2 \pi^{2} n_{I} v_{\mathbf{k}}}{\kappa_{I}}\left(\frac{2 m_{D} U_{0}}{\hbar^{2}}\right)^{2} \frac{1}{\left(4 k^{2}+\kappa_{I}^{2}\right)^{3 / 2}} .
$$

The contribution to the total relaxation rate (25) provided by the impurity scattering, $1 / \tau_{\mathrm{eff}}^{(I)}$, with (30) is calculated numerically.

Here, the parameter to be estimated is the inverse screening length $\kappa_{I}$. First, we can calculate $\kappa_{I}$ using the Thomas-Fermi

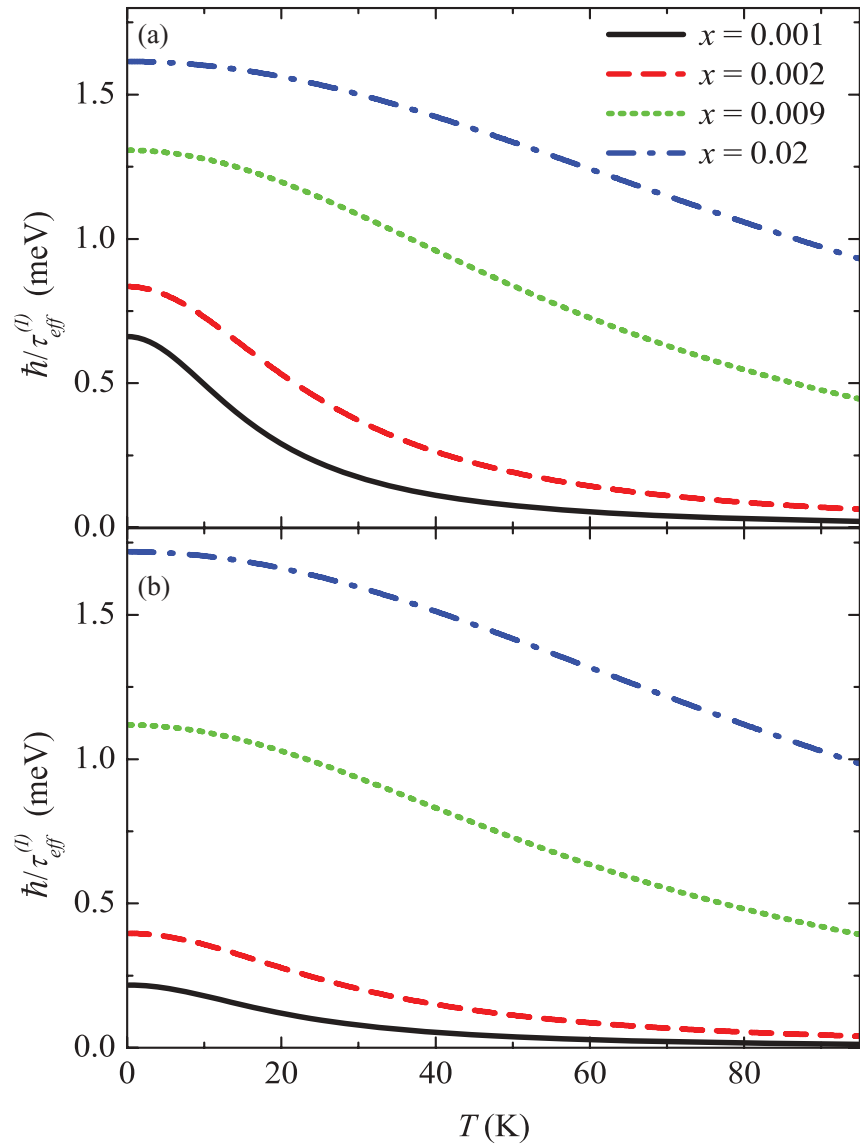

FIG. 2. (Color online) Residual relaxation rate provided by the scattering of the electrons on the impurities in $\mathrm{Nb}$-doped $\mathrm{SrTiO}_{3}$ as a function of the temperature (a) using the Thomas-Fermi screening length and (b) using the model screening length, the same for all samples.

(TF) approximation,

$$
\kappa_{I}^{(\mathrm{TF})}=\left(\frac{6 \pi n_{0} e^{2}}{\varepsilon_{0} E_{F}}\right)^{1 / 2},
$$

where $E_{F}=\left(\hbar^{2} / 2 m_{D}\right)\left(\pi^{2} n_{0}\right)^{2 / 3}$ is the Fermi energy for the threefold degenerate conduction band. We use here the static (rather than high-frequency) dielectric constant because the ions do not move, and hence the lattice polarization (provided by the polar LO phonons) reduces the Coulomb potential by the ratio $\varepsilon_{\infty} / \varepsilon_{0}$.

The results for the $1 / \tau_{\text {eff }}^{(I)}$ with (19) are shown in Fig. 2(a). The values of $1 / \tau_{\text {eff }}^{(I)}$ in the zero-temperature limit are in fair agreement with the experiment ${ }^{1}$ for the samples with two higher doping levels $x=0.9 \%$ and $x=2 \%$. However, for the two weaker doped samples, the residual relaxation rate calculated using (31) is overestimated with respect to the experiment. Therefore we can suggest that the spatial cutoff of the impurity potential can be provided also by additional mechanisms which can be irrelevant to the presence of the electron gas and which therefore do not vanish at low electron densities. In this connection, the contribution to the total relaxation rate due to the impurity scattering has been also calculated using the trial screening radius $r_{I} \equiv \kappa_{I}^{-1} \approx 0.76 \mathrm{~nm}$ 
which is one and the same for all samples and close to $\kappa_{I}^{(\mathrm{TF})}$ for the highest doping. The results for $1 / \tau_{\text {eff }}^{(I)}$ in this case are shown in Fig. 2(b). We see that in this case, the residual relaxation rate is in good agreement with the background for the total relaxation rate measured in the experiment. ${ }^{1}$ Moreover, the present result adequately reproduces the experimental density dependence of the residual relaxation rate without varying $\kappa_{I}$.

In our treatment of the impurity screening length, we suppose this quantity to be independent of doping in order to arrive at the experimentally observed trend. The assumption that $\kappa_{I}$ does not depend on the doping means that the dominant part of the impurity potential for the scattering processes is essentially short range. (Note that $r_{I} \approx 0.76 \mathrm{~nm}$ is about twice the lattice parameter.) The $\mathrm{Nb}$ ion is larger than the $\mathrm{Ti}$ atom and as a consequence the ions around the $\mathrm{Nb}$ donors are radially displaced. This has consequences for the local electronic structure and - as far as we know - nobody has analyzed this theoretically for $\mathrm{Nb}$ donors in $\mathrm{SrTiO}_{3}$. Empirically we know that even for a low $\mathrm{Nb}$ concentration, no bound donor states are formed. This is totally different from the case of Si doped with phosphor.

For the Yukawa potential (28), a critical value $\kappa_{I}^{(c)}$ exists such that for $\kappa_{I}>\kappa_{I}^{(c)}$, there are no bound states in that potential. The Monte Carlo calculation for a particle with an isotropic parabolic dispersion law ${ }^{20}$ gives

$$
\tilde{\kappa}_{I}^{(c)} \approx 1.1906 \tilde{U},
$$

where $\tilde{\kappa}_{I}^{(c)}$ and $\tilde{U}$ are, respectively, $\kappa_{I}^{(c)}$ and $U$ in the atomic units. We can estimate $\tilde{\kappa}_{I}^{(c)}$ for an anisotropic band using the parameters $\tilde{\kappa}_{I}^{(c)}$ and $\tilde{U}$ expressed in the effective atomic units for a crystal: $\tilde{\kappa}_{I}^{(c)}=\kappa_{I}^{(c)} a_{B}^{*}, \tilde{U}=U /\left(a_{B}^{*} E_{h}^{*}\right)$, with the effective Bohr radius $a_{B}^{*}=\varepsilon_{0} \hbar^{2} /\left(m_{D} e^{2}\right)$ and the effective Hartree energy $E_{h}^{*}=m_{D} e^{4}\left(\varepsilon_{0} \hbar\right)^{2}$. In Nb-doped strontium titanate, the critical inverse screening radius corresponding to (32) is $r_{I}^{(c)} \approx 2.5 \mathrm{~nm}$. The aforesaid value $r_{I} \approx 0.76 \mathrm{~nm}$ yields $\tilde{\kappa}_{I} \approx 3.9 \tilde{U}$, substantially larger than $\tilde{\kappa}_{I}^{(c)}$. Thus, in accordance with the aforesaid experimental evidence, there are no bound states in the Yukawa potential with the parameters of the $\mathrm{Nb}$-doped $\mathrm{SrTiO}_{3}$ chosen in the present calculation.

The other mechanisms which might contribute to the resistivity of $\operatorname{SrTi}_{1-x} \mathrm{Nb}_{x} \mathrm{O}_{3}$ are the direct scattering of the electrons by the optical phonons and the umklapp electronelectron scattering. These contributions have been separately calculated within the same kinetic equation approach as the aforesaid contributions due to the Baber and impurity scattering. In Fig. 3, the contribution to the relaxation rate due to the electron-LO-phonon scattering is plotted as a function of the temperature for the actual electron densities in the samples studied experimentally in Ref. 1. Under the experimental conditions of Ref. 1, the electron-LO-phonon scattering contribution to the relaxation rate appears to be relatively small with respect to the relaxation rate provided by the Baber mechanism. Furthermore, the relaxation rate due to the electron-LO-phonon scattering strongly decreases with decreasing temperature. Therefore the electron-phonon scattering is not one of the dominating mechanisms of the dc conductivity in Nb-doped $\mathrm{SrTiO}_{3}$.

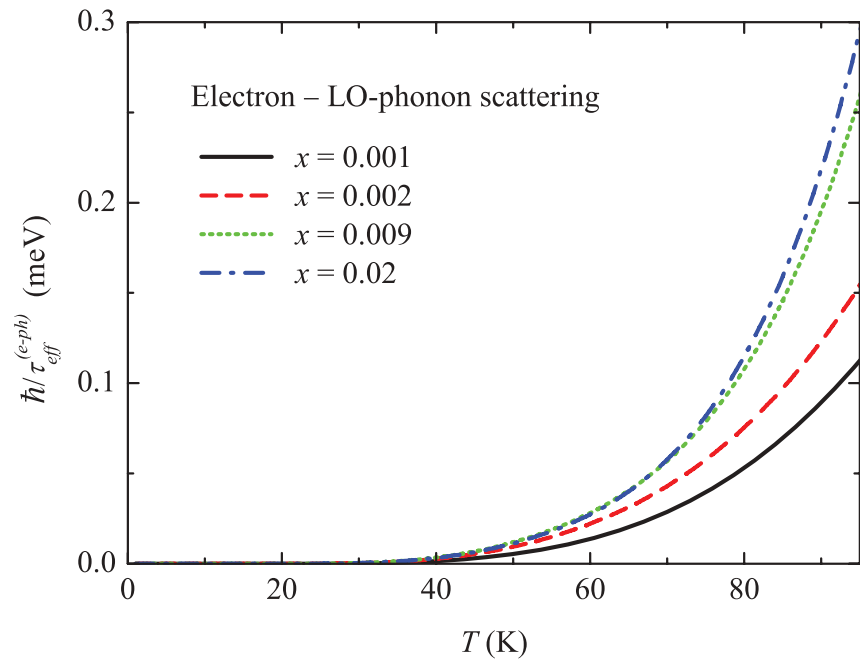

FIG. 3. (Color online) Contribution to the relaxation rate in $\mathrm{Nb}$ doped $\mathrm{SrTiO}_{3}$ due to the direct electron-LO-phonon scattering as a function of the temperature for the electron densities from Ref. 1.

Finally, the umklapp electron-electron scattering was considered in Ref. 1 as one of possible sources for the de resistivity in $\mathrm{Nb}$-doped $\mathrm{SrTiO}_{3}$. For the electron resistivity in metals, the Baber mechanism plays a minor role, and the umklapp scattering can be sufficient to explain the Fermi-liquid temperature dependence of the resistivity. ${ }^{5-7}$ However, the umklapp electron-electron scattering remarkably contributes to the resistivity only when both the initial and final momenta of the two electrons in the elementary scattering process lie in the thermal layer near the Fermi surface. Therefore, e.g. in the simple case of a spherically symmetric conductivity band, the umklapp scattering does not contribute to the resistivity when the maximal possible value of the momentum transfer $q_{\max }=4 \hbar k_{F}$ (where $k_{F}$ is the Fermi wave vector) is smaller than the modulus of the reciprocal lattice vector $\mathbf{g}$. Therefore the umklapp processes can be nonnegligible only at sufficiently high densities. For an anisotropic conductivity band with a warped Fermi surface (that is just the case in $\mathrm{SrTiO}_{3}$ ) the restriction for the density can be softened with respect to that for a spherically symmetric band. However, it is not $a$ priori known whether the umklapp scattering is relevant for the carrier densities in the experiment. ${ }^{1}$ Therefore we have estimated the umklapp contribution to the relaxation rate in strontium titanate within the kinetic equation approach.

In Fig. 4, the relaxation rate provided by the umklapp electron-electron scattering (multiplied by $\hbar / \mathcal{G}^{2}$, where $\mathcal{G}<1$ is the dimensionless interference factor for the umklapp processes ${ }^{14}$ ) is plotted as a function of the electron density for the temperature $T=80 \mathrm{~K}$. The relaxation rate provided by the umklapp processes in strontium titanate is not vanishingly small for densities higher than the threshold value $n_{0} \approx$ $8 \times 10^{20} \mathrm{~cm}^{-3}$. However, the carrier densities achieved in the experiment ${ }^{1}$ are substantially lower than this threshold value. In the figure, the arrows show the actual electron densities in the experiment. ${ }^{1}$ For the experimental densities $n_{0} \sim 10^{19} \mathrm{~cm}^{-3}$ to $10^{20} \mathrm{~cm}^{-3}$, the relaxation rate provided by the umklapp electron-electron scattering is vanishingly small with respect to the measured values of $1 / \tau$. The relaxation 


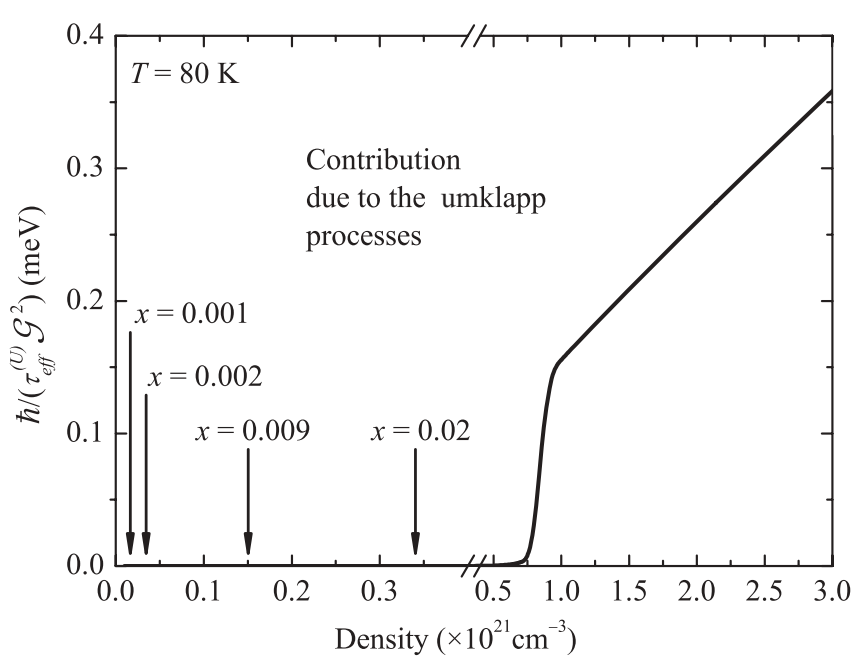

FIG. 4. Relaxation rate due to the umklapp electron-electron scattering in $\operatorname{SrTi}_{1-x} \mathrm{Nb}_{x} \mathrm{O}_{3}$ multiplied by $\hbar / \mathcal{G}^{2}$ [where $\mathcal{G}$ is the interference factor for the umklapp processes (Ref. 14)] as a function of the electron density for $T=80 \mathrm{~K}$. The arrows indicate the electron density for the samples of the experiment (Ref. 1). There are different scales at the $x$ axis for the densities $n_{0}<4 \times 10^{20} \mathrm{~cm}^{-3}$ and $n_{0}>4 \times 10^{20} \mathrm{~cm}^{-3}$.

rate due to the umklapp processes becomes nonnegligible in strontium titanate only for the electron densities $n_{0} \gtrsim$ $10^{22} \mathrm{~cm}^{-3}$, much higher than the densities relevant for the experiment. ${ }^{1}$ The conclusion follows that the contribution by umklapp processes to the Fermi-liquid behavior of relaxation rate and resistivity in $\mathrm{SrTi}_{1-x} \mathrm{Nb}_{x} \mathrm{O}_{3}$ is negligible. In summary, the electron-LO-phonon and umklapp scattering contributions to the dc resistivity of the $\mathrm{Nb}$-doped strontium titanate bring only relatively small corrections in the present treatment as far as it is related to the interpretation of the experiment. ${ }^{1}$

The relative contributions of different scattering mechanisms to the total relaxation rate as a function of temperature are shown in Fig. 5 for the sample with the doping content

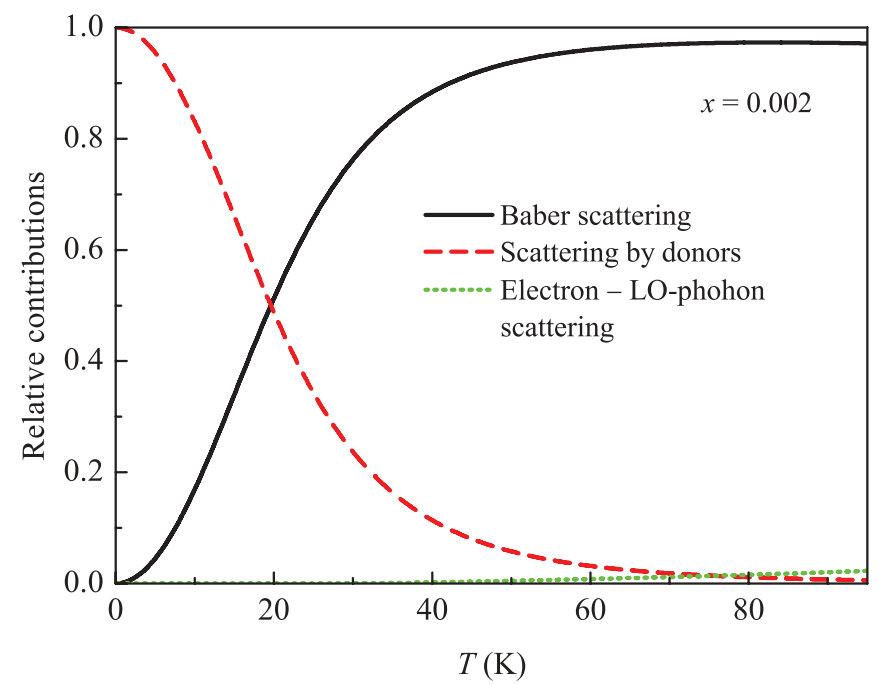

FIG. 5. (Color online) Relative contributions of different scattering mechanisms to the total relaxation rate as a function of temperature for the doping content $x=0.002$.

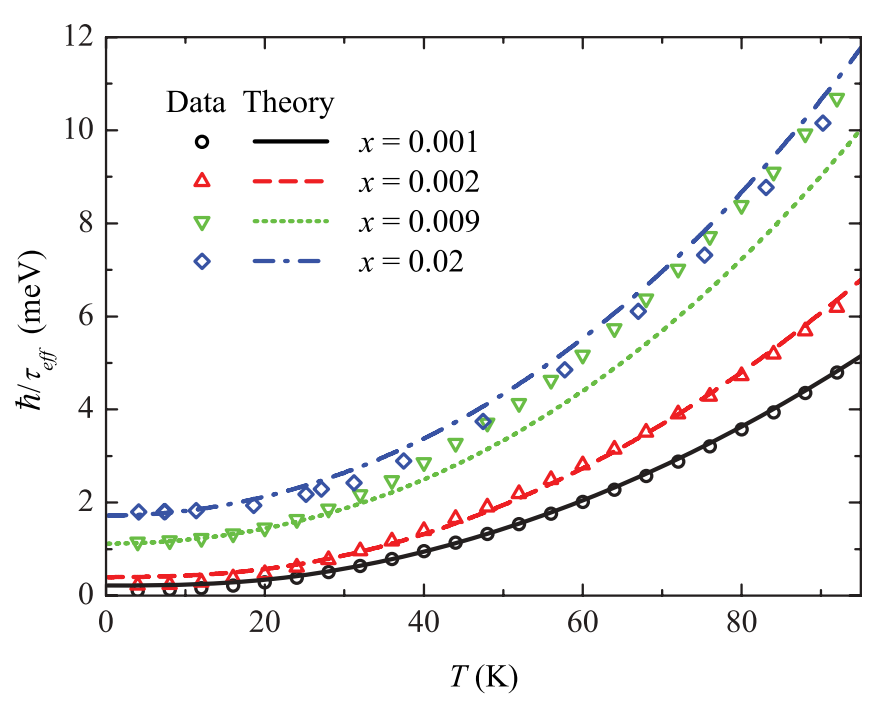

FIG. 6. (Color online) Curves: The calculated total relaxation rate in $\mathrm{Nb}$-doped $\mathrm{SrTiO}_{3}$ as a function of the temperature for the actual electron densities corresponding to the experimental values of the doping (Ref. 1). Symbols: Experimentally determined relaxation rate in $\mathrm{Nb}$-doped $\mathrm{SrTiO}_{3}$ from Fig. 2 of Ref. 1.

$x=0.002$. In the low-temperature range, the residual scattering of the electrons on the potential landscape of the impurities dominates. For higher temperatures, the Baber scattering plays a key role providing the Fermi-liquid $T^{2}$ dependence of the total relaxation rate. The electron-LO-phonon scattering only slightly contributes to the total relaxation rate and only at sufficiently high temperatures. Thus the main contribution to the relaxation rate is brought by the Baber scattering at moderate temperatures and by the scattering by the donors at low temperatures.

The total relaxation rate calculated taking into account the Baber, electron-impurity, and electron-LO-phonon scattering mechanisms is plotted in Fig. 6. The $T^{2}$ temperature dependence of the relaxation rate is completely due to the Baber mechanism. The scattering by donors is responsible for the residual relaxation rate which constitutes the background resistivity in $\mathrm{SrTi}_{1-x} \mathrm{Nb}_{x} \mathrm{O}_{3}$. We see that the aforesaid mechanisms convincingly explain both the temperature and density dependences for the experimentally measured relaxation rate.

\section{CONCLUSIONS}

In conclusion, the $\mathrm{dc}$ resistivity of $\mathrm{Nb}$-doped strontium titanate is explained in terms of two dominating mechanisms: Baber scattering with participation of both Coulomb and phonon-mediated electron-electron interactions provides the $T^{2}$ dependence of the resistivity and of the relaxation rate, while the scattering on the potential landscape caused by impurities yields the residual relaxation rate which does not vanish at $T=0$. The calculated relaxation rates are in a good agreement with the experiment. ${ }^{1}$ Thus the hypothesis on a common origin of two phenomena in $\mathrm{SrTiO}_{3}$ - superconductivity and the Fermi-liquid behavior of the resistivity - is supported by the microscopic calculations. 


\section{ACKNOWLEDGMENTS}

This work was supported by FWO-V projects G.0356.06, G.0370.09N, G.0180.09N, and G.0365.08, the WOG WO.035.04N (Belgium), the SNSF through Grant No.
200020-140761, and the National Center of Competence in Research (NCCR) "Materials with Novel Electronic Properties-MaNEP."
*On leave of absence from: Department of Theoretical Physics, State University of Moldova, str. A. Mateevici 60, MD-2009 Kishinev, Republic of Moldova.

${ }^{\dagger}$ Also at Lyman Laboratory of Physics, Harvard University, Cambridge, Massachusetts 02138, USA.

${ }^{1}$ D. van der Marel, J. L. M. van Mechelen, and I. I. Mazin, Phys. Rev. B 84, 205111 (2011).

${ }^{2}$ J. T. Devreese, S. N. Klimin, J. L. M. van Mechelen, and D. van der Marel, Phys. Rev. B 81, 125119 (2010).

${ }^{3}$ C. S. Koonce, M. L. Cohen, J. F. Schooley, W. R. Hosler, and E. R. Pfeiffer, Phys. Rev. 163, 380 (1967).

${ }^{4}$ N. Reyren, S. Thiel, A. D. Caviglia, L. F. Kourkoutis, G. Hammerl, C. Richter, C. W. Schneider, T. Kopp, A.-S. Ruetschi, D. Jaccard, M. Gabay, D. A. Muller, J.-M. Triscone, and J. Mannhart, Science 317, 1196 (2007).

${ }^{5}$ W. E. Lawrence and J. W. Wilkins, Phys. Rev. 7, 2317 (1973).

${ }^{6}$ A. H. MacDonald, Phys. Rev. Lett. 44, 489 (1980).

${ }^{7}$ A. H. MacDonald, R. Taylor, and D. J. W. Geldart, Phys. Rev. B 23, 2718 (1981).

${ }^{8}$ J. L. M. van Mechelen, D. van der Marel, C. Grimaldi, A. B. Kuzmenko, N. P. Armitage, N. Reyren, H. Hagemann, and I. I. Mazin, Phys. Rev. Lett. 100, 226403 (2008).
${ }^{9}$ J. Tempere and J. T. Devreese, Phys. Rev. B 64, 104504 (2001).

${ }^{10}$ G. M. Zhao, V. Smolyaninova, W. Prellier, and H. Keller, Phys. Rev. Lett. 84, 6086 (2000).

${ }^{11}$ H. Maebashi and H. Fukuyama, J. Phys. Soc. Jpn. 66, 3577 (1997).

${ }^{12}$ W. G. Baber, Proc. R. Soc. A 158, 383 (1937).

${ }^{13}$ T. Giamarchi and B. S. Shastry, Phys. Rev. B 46, 5528 (1992).

${ }^{14}$ J. M. Ziman, Electrons and Phonons (Oxford University Press, Oxford, 1960).

${ }^{15}$ G. D. Mahan, Many-Particle Physics, 2nd ed. (Plenum Press, New York, 1990).

${ }^{16}$ F. M. Peeters and J. T. Devreese, Phys. Rev. B 32, 3515 (1985).

${ }^{17}$ R. O. Bell and G. Rupprecht, Phys. Rev. 129, 90 (1963).

${ }^{18}$ A. I. Anselm, Introduction to Semiconductor Theory (Mir, Moscow/ Prentice-Hall, Englewood Cliffs, NJ, 1981).

${ }^{19}$ L. D. Landau and L. M. Lifshitz, Quantum Mechanics NonRelativistic Theory, Vol. 3 (Pergamon, Oxford, 1977).

${ }^{20}$ Y. Li, X. Luo, and H. Kröger, Sci. China G: Phys. Mech. Astr. 49, 60 (2006). 